\title{
Competição em floresta natural de araucária na região noroeste do Rio Grande do Sul-Brasil
}

\author{
Competition in natural Araucaria forest in northwestern \\ Rio Grande do Sul state, Brazil
}

\author{
Fernanda Raquel Lambrecht ${ }^{1}$, Rafaelo Balbinot ${ }^{2}$, Patricia Povoa de Mattos ${ }^{3}$, \\ Evaldo Muñoz Braz ${ }^{3}$, Jonathan William Trautenmüller ${ }^{4}$ e Aline Canetti ${ }^{4}$
}

\begin{abstract}
Resumo
Objetivou-se com o trabalho estimar, avaliar e selecionar o melhor índice de competição em um fragmento florestal na região noroeste do RS e analisar o comportamento da floresta pós manejo. O estudo foi realizado em

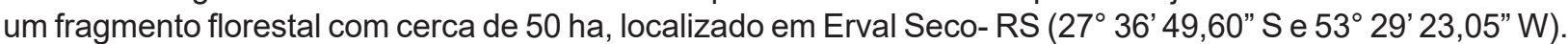
O fragmento apresenta vegetação classificada como ecótono, onde ocorre a transição da Floresta Ombrófila Mista (FOM) e Floresta Estacional Decidual (FED). Foram mensuradas 62 árvores de araucária (diâmetro a $1,30 \mathrm{~m}$ do solo $\geq 10 \mathrm{~cm}$ ). Para análise da competição da araucária foram analisados 13 índices, sendo eles dependentes da distância e independentes da distância. Dentre os 13 índices testados, os que apresentaram melhor desempenho foram IID9 (independente da distância) $\left(R_{a j}^{2}=0,7725 ; r=0,806\right.$; Sxy \% = 3,97) e IDD13 (dependente da distância) $\left(R_{\text {aj }}^{2}=0,6077 ; r=0,673 ; S x y \%=6,91\right)$. O incremento diamétrico dos indivíduos de araucária demostraram que para a espécie, após 20 anos de ter ocorrido o manejo florestal, há indicativo de que já poderia haver nova intervenção no local, pensando em favorecer os indivíduos jovens para a conservação dos mesmos e posteriormente buscando a produção de produtos madeireiros e não madeireiros.
\end{abstract}

Palavras-chave: Floresta Ombrófila Mista, Incremento, índices de competição.

\begin{abstract}
The objective of this work was to estimate, evaluate and select the best competition index in a forest fragment in the northwestern region of RS state and to analyze the behavior of the forest post - management. The study was carried out in a forest fragment with about 50 ha, located in Erval Seco, RS ( $27^{\circ} 36$ ' 49.60 "S and $53^{\circ} 29^{\prime}$ ' 23.05" W). The fragment shows vegetation classified as ecotone, where the transition from the Mixed Ombrophylous Forest and the Decidual Seasonal Forest occurs. 62 Araucaria trees (diameter at $1.30 \mathrm{~m}$ of soil $\geq 10 \mathrm{~cm}$ ) were measured. For analysis of the competition of the Araucaria 13 competition indexes were analyzed; both dependent of distance and independent of distance. Among the 13 tested indexes, the ones that presented the best performance were IID9 (distance independent) $\left(R^{2} a j=0.7725 ; r=0.806\right.$; Sxy\% $=3.97$ ) and IDD13 (distance dependent) $\left(R^{2} a j=0.6077 ; r=0.673 ; S x y \%=6.91\right)$. The diameter increase of Araucaria individuals showed that for the species, after 20 years of forest management, there is an indication that there could already be a new intervention in the area, in order to favor young individuals for conservation and later seeking the production of timber and non-timber products.
\end{abstract}

Keywords: Mixed Ombrophylous Forest, Increment, Competition indices.

\section{INTRODUÇÃO}

Em florestas naturais o manejo florestal em regime sustentado deve ser pensado como um conjunto de atividades que busque maximizar a produtividade que os recursos florestais nos proporcionam, mantendo foco nos aspectos ambientais, econômicos e sociais (HOSOKAWA, 1998).

\footnotetext{
1Departamento de Engenharia Florestal, Universidade Federal de Viçosa - UFV. Viçosa / MG, Brasil. e-mail: fernanda. lambrecht@hotmail.com

2Departamento de Engenharia Florestal, Universidade Federal de Santa Maria - UFSM. Santa Maria / RS, Brasil.

3Embrapa Florestas, Empresa Brasileira de Pesquisas Agropecuária - Embrapa. Colombo / PR, Brasil.

4Departamento de Engenharia Florestal, Universidade Federal do Paraná - UFPR. Curitiba / PR, Brasil.
}

Sci. For., Piracicaba, v. 47, n. 121, p. 131-138, mar. 2019 DOI: dx.doi.org/10.18671/scifor.v47n121.13 
Lambrecht et al. - Competição em floresta natural de araucária na região

noroeste do Rio Grande do Sul-Brasil

O manejo florestal é uma prática abordada que pode amenizar a competição entre os indivíduos. Quando a oferta dos recursos necessários para o crescimento das plantas decresce abaixo de sua demanda, a competição entre os indivíduos se inicia, e assim, afeta seu crescimento (WEBER et al., 2008).

Para realizar a quantificação do nível de competição entre árvores, podemos utilizar os índices de competição dependentes e independentes da distância. Esses índices podem avaliar o estado competitivo de uma árvore na floresta (CASTAGNERI et al., 2008), expressar o estresse imposto pela competição e representar a quantidade de recursos disponíveis para a árvore (EID; TUHUS, 2001). Podemos usar os índices para os modelos em nível de árvore individual (CRECENTE; MARSHALL; SOALLEIRO, 2009); em nível de floresta e em classes de tamanho (CAO, 1997; GONZÁLEZ et al., 2006).

Para realizar a modelagem de crescimento e produção em florestas naturais podemos fazer o uso dos índices de competição (FOLI, 1993) que podem permitir determinar o nível de supressão de cada árvore concorrente a árvore selecionada para ser mantida na floresta. (MARTINS et al., 2011). Segundo Stage (1973) muitas funções de crescimento úteis na previsão da taxa de crescimento de árvores individuais incluem variáveis que descrevem o estado da competição da árvore-objeto em relação às árvores circundantes.

Tendo em vista que o fragmento em estudo sofreu intervenção aos 20 anos, e que são poucos fragmentos em que se tem a oportunidade de analisar a resposta dessa intervenção, buscou-se levantar algumas questões: os índices de competição podem ou não indicar uma boa representação do comportamento dos indivíduos em floresta natural manejada? O manejo florestal em regime sustentado foi benéfico ou não para a Araucária?

Assim, o objetivo deste estudo foi ajustar, avaliar e selecionar o melhor índice de competição para fragmento em área de ecótono em Floresta Ombrófila Mista e Floresta Estacional Decidual, na região noroeste do Rio Grande do Sul e analisar o comportamento da floresta, pós manejo, realizado em 1993.

\section{MATERIAL E MÉTODOS}

Este estudo foi realizado em um fragmento florestal com cerca de 50 ha, localizado no município de Erval Seco (27 $36^{\prime} 49,60^{\prime \prime}$ S e $53^{\circ} 29^{\prime}$ 23,05” W), região noroeste do Rio Grande do Sul (RS). O fragmento em estudo apresenta vegetação classificada como ecótono, onde ocorre a transição da Floresta Ombrófila Mista e Floresta Estacional Decidual.

O clima da região é subtropical muito úmido, com regime de chuvas equilibrado, com média anual entre 1.700 e $1.900 \mathrm{~mm}$, contudo, há certa redução da precipitação durante o inverno. A temperatura média varia entre 20 e $23^{\circ} \mathrm{C}$ (ROSSATO, 2014).

Em 1993 foi realizado um Plano de Manejo Florestal em Regime Sustentado na área em estudo. O plano de manejo foi aprovado pela Secretaria Estadual do Meio Ambiente do Rio Grande do Sul. Após 20 anos, foi realizado outro inventário, em que, foram instaladas 31 parcelas de $20 \mathrm{~m}$ x $50 \mathrm{~m}$ totalizando uma área amostral de $1.000 \mathrm{~m}^{2}$ por parcela, em uma grade sistemática de $125 \mathrm{~m} \times 125 \mathrm{~m}$.

Nas 31 parcelas, foram medidas 62 árvores de araucária (consideradas árvores objeto). Foram identificadas as árvores concorrentes (competidoras), considerando aquelas cujas copas estavam em contato com a copa da árvore objeto. Nessas árvores foram mensuradas as seguintes variáveis: DAP; altura total; distância entre a árvore objeto e a competidora, posição sociológica e raio de copa.

O estado (status) competitivo de cada árvore objeto foi avaliado utilizando e comparando índices de competição, sendo que alguns são Índices Independentes da Distância (IID) e outros são Índices Dependentes da Distância (IDD) (Tabela 1). Os cálculos dos índices de competição, a correlação de Person entre DAP e índice de competição, coeficiente de determinação ajustado $\left(R_{\text {aj }}{ }\right)$, análise dos resíduos e o erro padrão da estimativa, foram realizados no Excel.

Foi realizada a análise dendrocronologica dos indivíduos de araucária, buscando ter os dados de incremento, para avaliar o comportamento ao longo do tempo, e qual a resposta de incremento da espécie em relação ao PMFS realizado em 1993. Buscando verificar a diferença significativa no incremento periódico anual (IPA 5anos) entre os períodos (1945-2015), foi realizado o teste de comparação de médias, com 99\% de confiabilidade. 
Tabela 1. Índices de competição utilizados, para analisar o comportamento da espécie Araucaria angustifolia submetida a manejo florestal em regime sustentado, na região noroeste do RS.

Table 1. Competition indexes used to analyze the behavior of the Araucaria angustifolia species submitted to sustainable forest management in northwestern RS state.

Índices independentes da distância (IID)

Glover e Hool (1979)

$$
I I D I=\frac{\pi\left(d_{i} / 2\right)^{2}}{\pi\left\{\left[\left(\sum_{i=I}^{n_{j}} d_{j}\right) / n_{j}\right] / 2\right\}^{2}}=\frac{d_{i}^{2}}{E Q \backslash x \backslash t o(D)^{2}}
$$

Daniels, Burkhart e Clason (1986) - Modificações do Índice de Glover e Hool (1979)

$$
\begin{gathered}
I I D 2=\frac{d_{i}{ }^{2}}{\left(\sum_{i=I}^{n} d_{j}{ }^{2}\right) / n} \\
I I D 3=\frac{h_{i}}{E Q \backslash x \backslash t o(H)} \\
I I D 4=\frac{d_{i}{ }^{2} \cdot h_{i}}{E Q \backslash x \backslash t o(D)^{2} \cdot E Q \backslash x \backslash t o(H)}
\end{gathered}
$$

Lorimer (1983)

$$
\text { IID5 }=\frac{\sum_{j=I}^{n} d_{j}}{d_{i}}=\sum_{j=1}^{n} \frac{d_{j}}{d_{i}}
$$

Corona e Ferrara (1989) - Modificação do índice de Lorimer (1983)

$$
\begin{gathered}
I I D 6=\frac{\sum_{j=I}^{n} d_{j}^{2}}{d_{i}{ }^{2}} \\
I I D 7=\frac{d_{i}}{d_{\max }}
\end{gathered}
$$

Tomé e Burkhart (1989), considerando o diâmetro máximo

BAL (Basal Area Larger), utilizado por Stage (1973)

$$
I I D 8=\sum_{i=1}^{n} g
$$

Stage (1973)

$$
I I D 9=\frac{d_{i}}{q}
$$

Índices dependentes da distância (IDD)

Hegyi (1974)

Martin e Ek (1984) - Modificação do índice de Hegyi

(1974) e de Daniels (1976).

$$
\begin{gathered}
\text { IIDI0 }=\sum_{j=1}^{n} \frac{d_{j}}{d_{i}} \cdot \frac{1}{L_{i j}} \\
\text { IIDII }=\sum_{j=1}^{n} \frac{d_{j}}{d_{i}} \cdot \frac{1}{\left(L_{i j}+1\right)} \\
\text { IID12 }=\sum_{j=1}^{n} \frac{h_{j}}{h_{i}} \cdot \frac{1}{L_{i j}} \\
\text { IDDI3 }=\sum_{j=1}^{n} \frac{d_{i}{ }^{2}}{d_{i}{ }^{2}+d_{j}{ }^{2}} \cdot L_{i j}
\end{gathered}
$$

Braathe (1980), citado/utilizado por Pukkala e Kolström (1987)

Moore et al. (1973) baseado no método de Brown (1965)

- Area Porentially Available (APA) - Área Potencialmente

Em que: $d_{i}$ e $d_{j}=$ diâmetro a altura do peito a $1,30 \mathrm{~m}$ de altura do solo (DAP) da árvore objeto $i$ e da árvore competidora $j(\mathrm{~cm})$, respectivamente; $=$ média dos diâmetros $(\mathrm{DAP})$ das árvores da unidade amostral $(\mathrm{cm}) ; n=$ número de árvores competidoras; $h_{i}$ e $h_{i}=$ altura total da árvore objeto $i$ e da árvore competidora $j(\mathrm{~m})$, respectivamente; = altura média das árvores da unidade amostral $(\mathrm{m}) ; q=$ diâmetro quadrático das competidoras $(\mathrm{cm}) ; \mathrm{g}=$ área transversal $\left(\mathrm{m}^{2}\right) ; d_{\max }=$ diâmetro a altura do peito a $\mathrm{I}, 30 \mathrm{~m}$ de altura do solo (DAP) máximo na unidade amostral $(\mathrm{cm}) ; \mathrm{L}_{\mathrm{ij}}=$ distância entre a árvore objeto e árvore competidora $(\mathrm{m})$. Fonte: adaptada de Curto (2015).

\section{RESULTADOS E DISCUSSÃO}

O DAP médio encontrado para os indivíduos de Araucária foi de $47 \mathrm{~cm}(11,7$ - 73,8 cm). A altura média encontrada foi de $20 \mathrm{~m}(7,5-26,5 \mathrm{~m})$. Os dados das árvores objeto e competidoras foram usados para o cálculo dos índices de competição. Testou-se 13 índices, e para analisar qual foi o melhor dentre os dependentes da distância e independentes da distância sendo analisados o coeficiente de determinação ajustado $\left(\mathrm{R}_{\text {aj) }}^{2}\right.$ correlação $(\mathrm{r})$ erro padrão da estimativa $\left(\mathrm{S}_{\mathrm{xy}} \%\right)$ e análise gráfica dos resíduos de cada índice de competição.

Dentre os 13 índices testados, os que apresentaram melhor desempenho foram IID9 (independente da distância) e IDD13 (dependente da distância). Na Tabela 2 são apresentadas as equações selecionadas e as estatísticas utilizadas para a seleção das melhores equações. 
Lambrecht et al. - Competição em floresta natural de araucária na região

noroeste do Rio Grande do Sul-Brasil

Tabela 2. Dados estatísticos da seleção de equações para a espécie Araucaria angustifolia, para os índices de competição, na região noroeste do RS.

Table 2. Statistical data of the selection of equations for the species Araucaria angustifolia, for the indices of competition in northwestern RS state.

\begin{tabular}{|c|c|c|c|c|c|}
\hline Índice & Autor & Equação & $\mathbf{R}^{2}{ }_{a j}$ & $\mathbf{r}$ & $\mathrm{S}_{\mathrm{xy}} \%$ \\
\hline IID1 & Glover e Hool (1979) & $y=0,0575 x-0,8158$ & 0,6276 & 0,792 & 5,25 \\
\hline IID2 & Daniels Burkhart e Clason (1986) & $y=0,0003 x^{2,2997}$ & 0,7636 & 0,698 & 8,50 \\
\hline IID3 & Daniels Burkhart e Clason (1986) & $y=0,0147 x+0,7156$ & 0,4109 & 0,641 & 2,79 \\
\hline IID4 & Daniels Burkhart e Clason (1986) & $y=0,0979 x-1,6119$ & 0,5587 & 0,747 & 6,50 \\
\hline IID5 & Lorimer (1983) & $y=-0,0251 x+4,367$ & 0,0546 & $-0,234$ & 7,31 \\
\hline IID6 & Corona e Ferrara (1989) & $y=-10,53 \ln (x)+43,979$ & 0,507 & $-0,626$ & 14,23 \\
\hline IID7 & Tomé e Burkhart (1989) & $y=0,0225 x-0,0276$ & 0,5119 & 0,716 & 4,77 \\
\hline IID8 & BAL (Basal Area Larger) & $y=-0,007 x+0,532$ & 0,2753 & $-0,525$ & 1,28 \\
\hline IID9 & Stage (1973) & $y=0,0171 x^{1,1499}$ & 0,7725 & 0,806 & 3,97 \\
\hline IID10 & Hegyi (1974) & $y=-0,0145 x+1,6324$ & 0,1149 & $-0,339$ & 9,50 \\
\hline IID11 & Martin e Ek (1984) & $y=-0,0415 x+6,1188$ & 0,081 & $-0,285$ & 7,51 \\
\hline IID12 & Braathe (1980), & $y=-0,0073 x+1,3823$ & 0,0591 & $-0,243$ & 6,29 \\
\hline IDD13 & Moore et al. (1973) & $y=0,0186 x^{1,6484}$ & 0,6077 & 0,673 & 6,91 \\
\hline
\end{tabular}

Nota: Coeficiente de determinação ajustado $\left(\mathrm{R}_{\mathrm{aj})}^{2}\right.$, correlação $(r)$, erro padrão $\left(\mathrm{S}_{\mathrm{xy}} \%\right)$.

O IID9 foi calculado a partir do DAP da árvore-objeto em relação ao diâmetro quadrático das competidoras, ou seja, foi uma relação entre os DAPs dos indivíduos que estão competindo com a araucária (Figura 1). O IDD13 expressa a competição exercida por árvores circundantes relacionando DAP e distância (Figura 2). O uso do DAP foi importante nesse caso, pois queremos definir quais diâmetros estão com maior pressão de competição. Esse entendimento é decisivo para quem for realizar um manejo florestal.

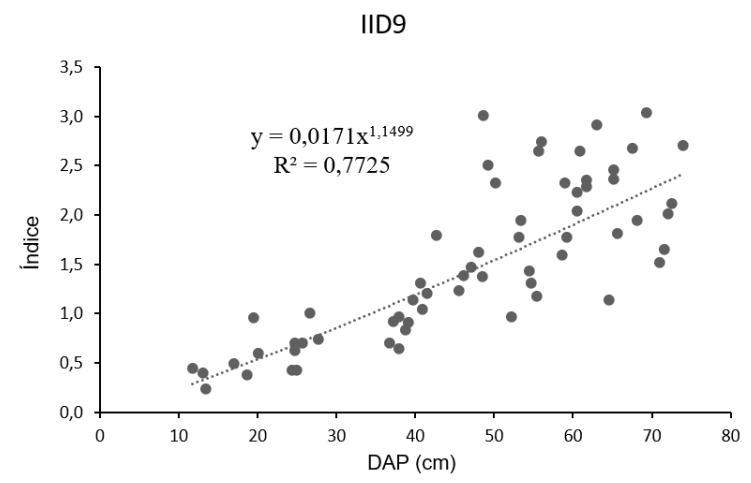

Figura 1. Relação entre índice de Stage (1973) (IID9) e o DAP realizado para a Araucaria angustifolia e suas competidoras, na região noroeste do RS.

Figure 1. Relationship between Stage index (1973) (IID9) and DBH realized for Araucaria angustifolia and its competitors, in northwestern RS state.

IDD13

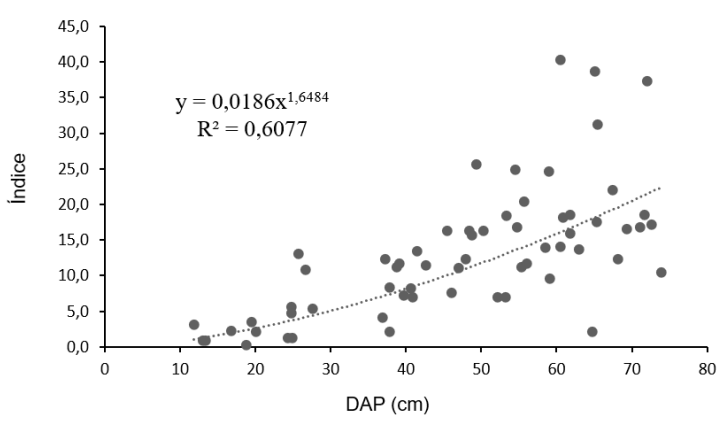

Figura 2. Relação entre o índice de Moore et al. (1973) (IDD13) e o DAP realizado para a Araucaria angustifolia e suas competidoras, na região noroeste do RS.

Figure 2. Relationship between the Moore et al. index (1973) (IDD13) and the DBH for Araucaria angustifolia and its competitors, in northwestern RS state. 
O índice de competição expressa a competição das árvores vizinhas (árvores que apresentam influência direta no espaço vital de cada árvore objeto), a medida que esse ambiente for comprometido pelo crescimento e ocupação do espaço por árvores concorrentes (vizinhas). Nesses casos, quanto menor o valor do índice, maior a competição.

Verificou-se que a fórmula define os diâmetros das competidoras no denominador (Tabela 1). Portanto, quanto maior o diâmetro das competidoras, menor será o valor do índice (e maior a competição pois a relação é inversa). Se o valor do índice aumenta, quer dizer que a medida que a árvore cresce, ela conseguirá superar as barreiras da competição. Essa análise pode ser feita para os índices IID9 e IDD13 pois respondem de forma inversa.

Curto (2015) estudando um plantio superestocado de Araucária encontrou como respostas dos melhores índices, os mesmos do presente estudo, sendo Stage $(1973)\left(R^{2} a j=0,90 ; S y x \%=13,59\right)$ e Moore et al. (1973) $\left(R^{2} a j=0,81 ; S y x \%=19,07\right)$. Seus valores foram diferenciados, pois Curto (2015) obteve informações de um plantio de Araucária superestocado, enquanto que o presente trabalho estudou um fragmento florestal natural que foi manejado.

Ricken (2014) realizando trabalho no município de Lages com a espécie araucária encontrou uma correlação negativa entre o aumento dos índices de competição e o Incremento Periódico Anual em diâmetro. Com isso Ricken (2014) observou que quanto maior a competição entre os indivíduos, menor será o incremento da árvore. Cunha (2013) encontrou também correlação negativa para as espécies Cedrela odorata, Amburana cearensis, Copaifera paupera e Swietenia macrophylla, em estudos dos índices de competição com o incremento periódico em área basal. Os efeitos da competição sobre o crescimento das árvores-objeto denotam que tratamentos silviculturais e manejo adequado são essenciais para o rendimento da floresta e, consequentemente, aumento dos componentes dendrométricos, favorecendo o crescimento sustentável da produção e recursos do ambiente (RICKEN, 2014).

Os resultados do presente estudo, mesmo sendo em transição entre FOM e FED, foram semelhantes, sendo observado que indivíduos de maior tamanho apresentavam maiores taxas de crescimento e, ao longo do tempo, menor competição. Castro (2012) verificou estas afirmativas ao estudar o comportamento médio de crescimento (diâmetro e altura) e a probabilidade de mortalidade para todo o conjunto de árvores de um fragmento de Floresta Estacional Semidecidual Montana em Viçosa, MG.

Rossi et al. (2007) afirmaram que as árvores, de diferentes espécies morrem em razão da influência direta da sua capacidade de competir com outras em uma idade específica, independentemente do local em que elas estejam. Isso justifica o uso, na maioria das vezes, do índice de competição nos modelos de mortalidade para árvores individual (CRECENTE; MARSHALL; SOALLEIRO, 2009).

Ricken (2014) observou que quanto maior a distância entre os indivíduos, maior será a área de copa e, consequentemente, também maior o incremento periódico em diâmetro. Assim, menor distância resulta em maior competição, o que prejudica a formação da copa, o incremento e o crescimento da floresta. Esse resultado pode ser entendido quando se menciona Larson (1963) o qual defende que em povoamentos fechados as árvores dominadas vão sendo sombreadas e o aumento progressivo dessa competição provoca a redução no tamanho de sua copa, o que resulta em declínio na produção de madeira, assim como em mudanças na distribuição do crescimento ao longo do fuste e na largura dos anéis de crescimento.

A tendência de comportamento dos indivíduos de Araucária ao longo dos anos (IPA 5 anos), distribuídos em três classes diamétricas, está apresentado na figura 3. Foi observado indicativo que os indivíduos mais jovens ( $1^{\text {a }}$ classe:10-24,9) apresentam incremento maior em relação as demais. Foi possível notar incremento crescente nos anos de 1950 a 1970 (classe 3), seguido de decréscimos ao longo dos anos, demostrando um indicativo de intervenção nesse momento no fragmento. Não foi possível determinar qual a quantidade de madeira retirada na época, porém houve uma tendência de resposta de incremento crescente para os indivíduos $2^{\mathrm{a}}$ classe, nesse período.

Em 1993, houve o PMFS aprovado pela secretaria estadual do meio ambiente. Nessa fase verificou-se uma diminuição no incremento ( $2^{\mathrm{a}}$ classe e $3^{\mathrm{a}}$ classe), até 2005 onde aparece um leve pico para a $3^{\text {a }}$ classe. A partir de 2010 o incremento tende a diminuir indicando que poderia ser o momento de realizar uma nova intervenção (novo manejo florestal em regime sustentado) (Figura 3).

Realizou-se a análise do incremento periódico anual (IPA 5 anos) ao longo do tempo (1945-2015) e verificou-se que houve diferença entre IPA ( 5 anos), observando que o período de maior incremento foi o IPA em 1965 e 1975. Os novos picos em 1980, 2000 e 2005, não diferiram entre si (Figura 4). 
Lambrecht et al. - Competição em floresta natural de araucária na região

noroeste do Rio Grande do Sul-Brasil

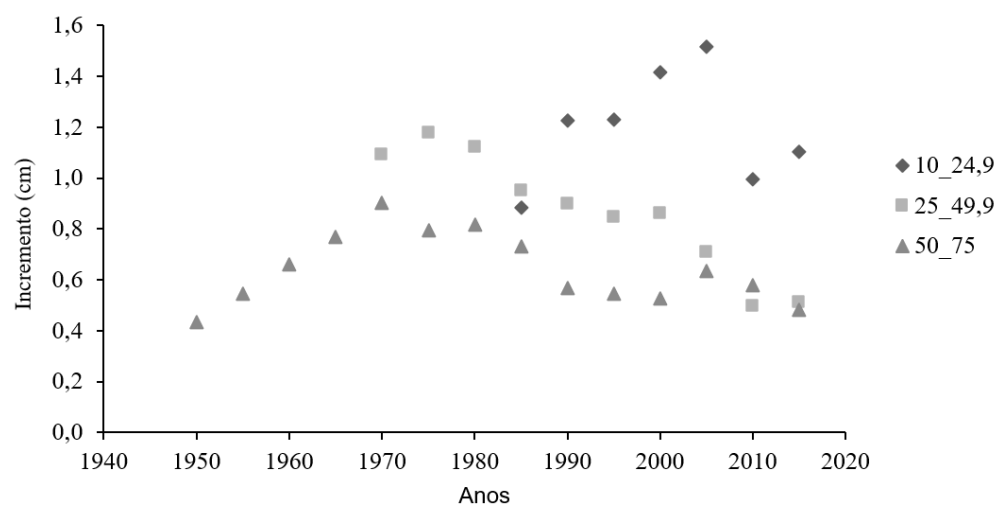

Figura 3. Incremento periódico anual (IPA 5 anos) ao longo do tempo, distribuídos em três classes diamétricas, observando o comportamento da Araucaria angustifolia (Bertol) Kuntze ao longo do tempo, na região noroeste do RS.

Figure 3. Annual periodic increment (PAI 5 years) over time, distributed in three diametric classes, observing the behavior of Araucaria angustifolia (Bertol) Kuntze over time, in northwestern RS state.

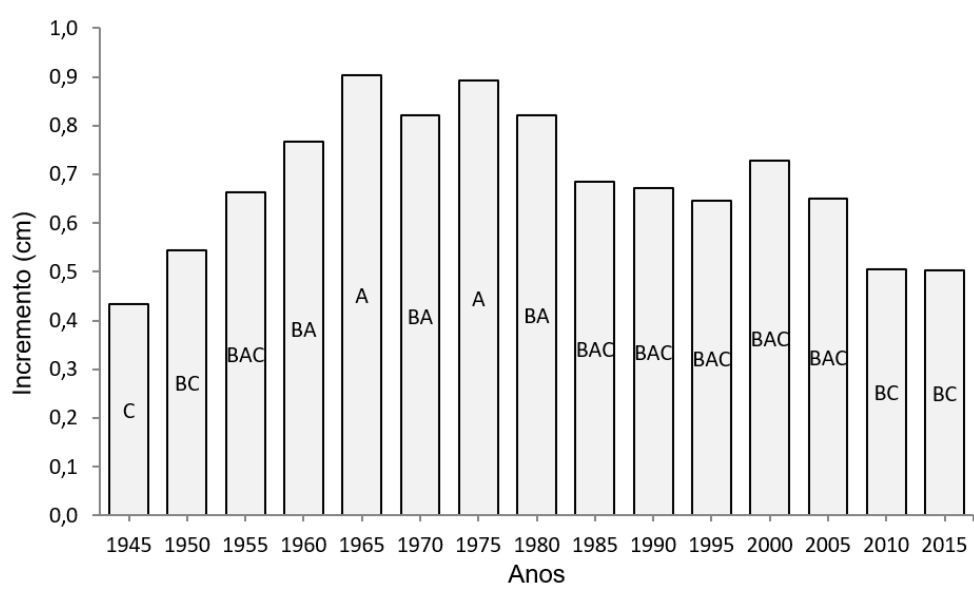

Figura 4. Incremento periódico anual em relação a idade de Araucaria angustifolia (Bertol) Kuntze, na região noroeste do RS.

Figure 4. Annual periodic increment in relation to the age of Araucaria angustifolia (Bertol) Kuntze, in northwestern RS state.

Estudos com outras espécies, ex: Pinus (ANDRADE et al. 2007) indicam que árvores dominadas apresentam maior crescimento na parte superior do fuste, enquanto que em árvores livres de concorrência o maior crescimento em área transversal foi registrado na base da árvore (ANDRADE et al., 2007; ASSMANN, 1970). O tamanho das árvores vizinhas foi considerado por Hiley (1959) e Potvin; Dutilleul (2009) como um dos fatores mais importantes para limitação do crescimento de árvores em um mesmo povoamento.

Curto (2015) notou em seu estudo com araucária que a árvore de maior classe de diâmetro recuperou ao longo do seu ciclo de desenvolvimento um padrão de crescimento mais regular;por outro lado, a árvore da menor classe apresentou distorções evidentes de crescimento ao longo do fuste, indicando variações em função da pressão acarretada pela alta competição.

Os índices de competição são uma alternativa simples e barata que pode auxiliar o manejador na tomada de decisão, para avaliação de quais indivíduos de interesse estão sofrendo maior pressão e podem ser liberados dessa competição, para futura extração.

\section{CONCLUSÕES}

Os índices de competição que apresentaram melhores resultados foram o índice de Stage (1973) (IID9), e dentre os índices dependentes da distância (IDD) foi o de Moore et al. (1973) (IDD13); estes são uma forma simples e de baixo custo para analisar os efeitos da competição sobre os indivíduos, porém é bom lembrar, que apresentam suas limitações. 
Os dados de incremento demostram que após os 20 anos do manejo florestal, tem indícios que já poderia haver nova intervenção, pensando em favorecer os indivíduos jovens remanescente e buscando a conservação da Araucária, para que na sequência possa ser realizado intervenção de cunho para produção.

\section{REFERÊNCIAS BIBLIOGRÁFICAS}

ANDRADE, C. M.; FINGER, C. A. G.; THOMAS, C.; SCHNEIDER, P. R. Variação do incremento anual ao longo do fuste de Pinus taeda L. em diferentes idades e densidades populacionais. Ciência Florestal, Santa Maria, v. 17, n. 3, p. 239-246, 2007.

ASSMANN, E. The principles of Forest Yeld Study: studies in the organic production, structure, increment and yeld of forestas stands. Oxford: Pergamon Press. 1970. 506 p.

BRAATHE, P. Height increment of young single trees in relation to height and distance of neighbouring trees, Mitt Forstl. VersAnst. v. 130, p. 43-48, 1980.

BROWN, G. S. Point density in stems per acre. Wellington: Forest Research Institute, 1965. 11 p. (New Zealand Forest Service n. 38)

CAO, Q.V. A method to distribute mortality in diameter distribution models. Forest Science, v. 43, p. 435442, 1997.

CASTAGNERI, D.; VACCHIANO, G.; LINGUA, E.; MOTTA, R. Analysis of intraspecific competition in two subalpine Norway spruce (Picea abies (L.) Karst.) stands in Paneveggio (Trento, Italy). Forest Ecology and Management, Amsterdam, v. 255, p. 651-659, 2008.

CASTRO, R V O. Modeling individual tree for an Semideciduous Forest using neural network. 2012. 105 p. Tese (Doutorado em Ciência Florestal) - Universidade Federal de Viçosa,Viçosa, 2012.

CORONA, P.; FERRARA, A. Individual Competition Indices for Conifer Plantations. Agriculture, Ecosystems and Environment, v. 27, p. 429-437, 1989.

CRECENTE, F C..; MARSHALL, P.; SOALLEIRO, R. Modeling non catastrophic individual tree mortality for Pinus radiata plantations in Northwestern Spain. Forest Ecology and Management, Amsterdam, v.257, p.1542-1550, 2009.

CUNHA, T. A.; FINGER, C. A. G. Competição assimétrica e o incremento diamétricode árvores individuais de Cedrela odorata L. na Amazônia ocidental. Acta Amazônica, Manaus, v. 43, n. 1, p. 9-18, 2013.

CURTO, R. A. Avaliação do crescimento e potencial de manejo em plantio superestocado de Araucaria angustifolia (Bert.) O. Ktze. 2015. 250 p. Tese (Doutorado em Engenharia Florestal) - Universidade Federal do Paraná, Curitiba, 2015.

DANIELS, R. F. Simple Competition Indices and Their Correlation with Annual Loblolly Pine Tree Growth. Forest Science, v. 22, n. 4, p. 454-456, 1976.

DANIELS, R. F.; BURKHART, H. E.; CLASON, T. R. A comparison of competition measures for predicting growth of loblolly pine trees. Canadian Journal Forest Research, Otawa, v. 16, p. 1230-1237, 1986.

EID, T.; TUHUS, E. Models for individual tree mortality in Norway. Forest Ecology and Management, Amsterdam, v. 154, n. 1, p. 69-84, 2001.

FOLI, E.G. Crown dimensions and diameter growth of some tropical mixedforest tree s in Ghana. 1993. 185 p. Tese (Doutorado) - University of Aberdeen, Aberdeen, 1993.

GLOVER, G. R.; HOOL, J. N. A basal area ratio predictor of loblolly pine plantation mortality. Forest Science, v. 25 , n. 2, p. 275-282, 1979.

GONZÁLEZ, M. S.; RÍO, M.; CAÑELLAS, I.; MONTERO, G. Distance independent tree diameter growth model for cork oak stands. Forest Ecology and Management, Amsterdan, v. 225, p. 262-270, 2006.

Sci. For., Piracicaba, v. 47, n. 121, p. 131-138, mar. 2019

DOI: dx.doi.org/10.18671/scifor.v47n121.13 
Lambrecht et al. - Competição em floresta natural de araucária na região

noroeste do Rio Grande do Sul-Brasil

HEGYI, F. A simulation model for managing jack-pine stands. In: FRIES, J. Growth models for tree and stand simulation. Stockholm Sweden: Royal College of Forestry, 1974. p. 74-90.

HILEY, W. E. Conifers: South African methods of cultivation. London: Faber and Faber, 1959. 123 p.

HOSOKAWA, R. T.; MOURA, J. B.; CUNHA, U. S. Introdução ao manejo e economia de florestas. Curitiba: Editora da UFPR, 1998.

LARSON P. R. The Vascular Cambium: Development and Structure. Berlim: Springer- Verlag, 1994.

LORIMER, C. G. Tests of age-independent competition indices for individual trees in natural hardwood stands. Forest Ecology and Management, Amsterdam, v. 6, p. 343-360, 1983.

MARTIN, G. L.; EK, A. R. A comparison of competition measures and growth models for predicting plantation red pine diameter and height growth. Forest Science, v. 30, n. 3, p. 731-743, 1984.

MARTINS, F.B.; SOARES, C.P.B.; LEITE, H.G.; SOUZA, A.L.; CASTRO, R.V.O.Índices de competição em árvores individuais de eucalipto. Pesquisa Agropecuária Brasileira, Brasília, v.4, n. 6, p.1089-1098, 2011.

MOORE, J. A.; BUDELSKY, C. A.; SCHLESINGER, R. C. A New Index Representing Individual Tree Competitive Status. Canadian Journal Forest Research, Otawa, v. 3, n. 4, p. 495-500, 1973.

POTVIN, C.; DUTILLEUL, P. Neighborhood effects and size-asymmetric competition in a tree plantation varying in diversity. Ecological Society of America, v. 90, n. 2, p. 321-327, 2009.

PUKKALA, T.; KOLSTROM, T. Competition indices and the prediction of radial growth in Scots pine. Silva Fennica, v. 21, n. 1, p. 55-67, 1987.

RICKEN, P. Incremento, espaço horizontal e competição em povoamento natural de Araucaria angustifolia no planalto catarinense. 2014. 105 p. Dissertação (Mestrado em Engenharia Florestal) - Universidade do Estado de Santa Catarina. Programa, Lages, 2014.

ROSSATO, M. S. Os climas do Rio Grande do Sul: Tendências e tipologias. In: MENDONÇA, F. (Org.). Os climas do Sul: Em tempos de mudanças climáticas globais. Jundiaí: Paco Editorial, 2014. p. 217-271.

ROSSI, L. M. B.; KOEHLER, H. S.; SANQUETTA, C. R.; ARCE, J. E. Modelagem da mortalidade em florestas naturais. Revista Floresta, Curitiba, v. 37, p. 275-291, 2007.

STAGE, A. R. Prognosis model for stand development. Madison: USDA Forest Service, 1973. (Research Paper INT-137)

TOMÉ, M.; BURKHART, H. E. Distance-dependent competition measures for predicting growth of individual trees. Forest Science, v. 35, n. 3, p. 816-831, 1989.

WEBER, P.; BUGMANN, H.; FONTI, P.; RIGLING, A. Using a retrospective dynamic competition index to reconstruct forest succession. Forest Ecology and Management, Amsterdam, v. 254, p. 96-106, 2008.

Recebido em: 22/02/2018

Aceito em: 08/08/2018 